\title{
IMPACTO DE LA RESPONSABILIDAD SOCIAL Y EL CAPITAL INTELECTUAL EN LA COMPETITIVIDAD DE LAS PYMES MANUFACTURERAS DE AGUASCALIENTES
}

\author{
IMPACT OF SOCIAL RESPONSIBILITY AND INTELLECTUAL \\ CAPITAL IN COMPETITIVENESS OF MANUFACTURING SMES \\ OF AGUASCALIENTES
}

Luis Aguilera Enríquez Universidad Autónoma de Aguascalientes, México laguiler@correo.uaa.mx
Héctor Cuevas Vargas Universidad Autónoma de Aguascalientes, México hcuevas@utsoe.edu.mx
Octavio Hernández

Castorena

Universidad Autónoma de Aguascalientes, México ohernandez@correo.uaa.mx

\section{RESUMEN}

El presente estudio empírico de tipo explicativo tiene como objetivo identificar el impacto de las variables responsabilidad social y capital intelectual en la competitividad de las pequeñas y medianas empresas (PYMES) manufactureras de Aguascalientes. El instrumento utilizado fue un cuestionario, el cual se sometió a una prueba de fiabilidad estadística y se obtuvo un .962 en el coeficiente Alpha de Cronbach, que al ser aplicado a los gerentes de 150 PYMES manufactureras los resultados obtenidos, a través del análisis de regresión lineal múltiple bajo el método de pasos sucesivos, permiten inferir que tanto la variable responsabilidad social como el capital intelectual influyen de manera positiva en la competitividad de las PYMES manufactureras de Aguascalientes a un nivel de significancia de 0.001 y juntas explican la competitividad en 57.8 por ciento.

Palabras clave: competitividad, PYME manufacturera, responsabilidad social, capital intelectual.

Clasificación JEL: M14, O15, O34, P47. 


\begin{abstract}
This empirical study of correlational type was aimed to identify the impact of the variables social responsibility and intellectual capital in the Competitiveness of Manufacturing Small and Medium Enterprises (SMEs) of Aguascalientes. The instrument used was a questionnaire which was submitted to a test of statistical reliability which got a 962 Cronbach's alpha coefficient, and which have been applied to the managers of 150 manufacturing SMEs, the results obtained by Linear Regression Analysis under the Successive Steps method infer that both the variable Social Responsibility and Intellectual Capital impact positively in the Competitiveness of manufacturing SMEs in Aguascalientes to a significance level of 0.001, which together explain in a $57 \%$ the competitiveness.
\end{abstract}

Key words: competitiveness, manufacturing SME's, social responsibility, intellectual capital. 


\section{INTRODUCCIÓN}

En la actualidad, el crecimiento y desempeño de la pequeña y mediana empresa (PYME) ha sido constante en todos sus sectores y han tratado de no descuidar el rendimiento de las áreas funcionales de este tipo de organizaciones. En este sentido, las empresas se han preocupado por mejorar un aspecto relevante como lo es la responsabilidad social (Ussahawanitchakit, 2005; Winn et al., 2010), es por eso que las empresas requieren tener la habilidad de resolver problemas que generalmente se gestionan dentro de las áreas funcionales (Ussahawanitchakit, 2005; Bibri, 2008). La responsabilidad social (RS) influye de manera significativa en cómo elevar los índices de competitividad empresarial (Weber, 2008), para ello es importante mejorar constantemente la motivación con los empleados, y desde luego los servicios con los clientes (Smith, 2003).

La RS, desde un punto de vista esencial y conceptual, no deja de tener influencia relevante en los objetivos que tienen los empresarios y líderes en las organizaciones donde el factor competitividad es un elemento meta que no debe perderse de vista en el desarrollo de las empresas, como es el caso de la PYME (van de Ven y Jeurissen, 2005; Porter y Kramer, 2006). Es cierto que la posición del mercado permite a las empresas analizar su nivel de competitividad, razón por la cual la relación que tenga la RS y la competitividad debe ser objetiva y eficaz para que elementos clave como las finanzas, la actividad operativa y la relación entre el personal en todos los niveles permita a las empresas tener resultados atractivos, acorde a las metas y valores corporativos propuestos por los empresarios (Wernerfelt, 1984; Van de Ven y Jeurissen, 2005; Jenkins, 2009).

Para el caso concreto de la PYME manufacturera, la RS ha sido de gran apoyo para que la relación de negocios se dé con otras organizaciones 
sin problemas, en especial porque el personal en todos sus niveles entiende que se deben trabajar con más sentido aspectos como la ideología, las políticas y los buenos ambientes (Bagdoniene y Paulaviciene, 2010). Para ello es importante resaltar que, al darle mayor enfoque a estos elementos, la PYME tendrá mayores beneficios sociales por su influencia en aspectos como el medio ambiente y en los aspectos sociales necesarios en las organizaciones (Avram y Kühne 2008; Jenkins, 2009). Por otro lado, la aportación a la sociedad por parte de la PYME permite promover el crecimiento económico, aumentar el empleo, promover la mejora tecnológica y mantener la armonía interna entre los empleados (Bagdoniene y Paulaviciene, 2010).

Asimismo, para las organizaciones en general, el capital intelectual (CI) en la PYME manufacturera es visto como un factor importante a considerar dentro de las estrategias de crecimiento, a pesar de su intangibilidad para formar parte de los recursos que deben administrarse por parte de los líderes y empresarios (Bounfour, 2003; Ross, Pike y Fernstrom, 2005). Por tal motivo, el CI debe formar parte de las estrategias competitivas que permitan a las organizaciones tener presencia en los mercados y ser realmente significativas al pensar en que la administración de los recursos deben estar fortalecida al aprovechar al máximo las bondades que implica dentro de las empresas el darle importancia y desarrollo al CI (Szymura-Tyc, 2009). Es importante resaltar que para la PYME manufacturera el control de los recursos es elemental para su supervivencia pero controlar el recurso humano depende de varios factores para que su beneficio sea óptimo, eficaz y permanente (Vázquez, Sánchez y Rodríguez, 2012).

En la actualidad ha sido importante en la PYME manufacturera poner atención en los cambios que se han presentado en la economía global, además de darle importancia a las tendencias locales de cómo administrar una organización como la PYME al tomar en cuenta lo complejo de cada 
una de sus áreas funcionales internas (Lipovatz, Mandaraka y Mourelatos, 2000). En este sentido, el aprovechamiento del CI permitirá a dicho tipo de empresas tener un importante crecimiento y desarrollo en áreas claves como la administración básica, las finanzas, el suministro de los recursos materiales, los procesos productivos y las gestiones de ventas; desde luego que el CI debe beneficiar a las organizaciones en contar con elementos que puedan hacer buen uso de las estrategias necesarias que les permita cumplir con sus metas y objetivos de competitividad y desempeño (Beyers, 2006; Bryson, Taylor y Cooper, 2008).

El presente análisis se ha propuesto como objetivo el identificar si los elementos contenidos en la responsabilidad social y el capital intelectual son factores clave para poder tener una mayor competitividad en la PYME manufacturera. Para ello es importante que se cuestione, por un lado, si la responsabilidad social es básica para que se tenga una mayor competitividad en la PYME manufacturera y, por otro, si el capital intelectual permite que las PYME manufactureras sean más competitivas. El trabajo de investigación se realizó en el estado de Aguascalientes con una muestra de 150 empresas y encuestas aplicadas a los gerentes en un periodo entre septiembre y noviembre del año 2012 y se divide en seis secciones; después de la introducción se revisan la literatura y las hipótesis formuladas; la tercera sección comprende la metodología; en la cuarta se presentan el análisis y resultados de la investigación; la quinta sección contiene la discusión; para finalizar, la última está compuesta por las conclusiones, limitaciones del estudio y las futuras líneas de investigación. 


\section{REVISIÓN DE LA LITERATURA}

El modelo de investigación que aquí se desarrolla describe la relación de la responsabilidad social y el capital intelectual en la competitividad de las PYMES. Las siguientes secciones buscan clarificar los distintos componentes del modelo objeto de estudio con la intención de sustentar los planteamientos y resultados obtenidos.

\subsection{Responsabilidad social y la competitividad en la PYME manufacturera}

Hoy en día la pequeña y mediana empresa, PYME, está interesada en el tema de la responsabilidad social (RS), ya que se pueden atender con facilidad aspectos sociales, laborales y ambientales con un enfoque específico de mejorar el bienestar de los trabajadores (Avram y Kühne, 2008), tema en donde se puede observar que la PYME, en especial la manufacturera, tienen algunas limitaciones que en ocasiones detienen el desarrollo y crecimiento de las organizaciones, porque en el tema empresarial: la gestión de los recursos financieros, el control de los apoyos externos o el adecuado manejo de las habilidades de los empresarios y líderes deben estar enfocados a que la sociedad en general vea a este tipo de organizaciones comprometidas con la RS (Cepinskis, Kvedaravicius y Zigutis, 2005; Jenkins, 2009; Laurinkeviciute y Stasiskiene 2010).

El interés que se ha tenido hasta ahora por darle mayor auge al tema de la responsabilidad social por parte de los gobiernos, instituciones y algunas organizaciones, en especial las que operan sin fines de lucro, permite que se profundice sobre los resultados que hasta ahora han tenido este tipo de organizaciones. Al tener como referencia experiencias pasadas 
sobre el desarrollo y aprovechamiento de los recursos, los mismos organismos analizan desde un punto de vista ético y de RS lo importante que es el desarrollo de prácticas de mejora de manera que se vean importantes beneficios tanto en los lugares de trabajo como en los aspectos sociales, al margen de las obligaciones que deben tener las organizaciones con la sociedad (Vogel, 2005).

Es importante resaltar que, en la actualidad, la participación en la economía de las regiones es muy importante, y es por ello que la RS al integrarse a la actividad laboral de este tipo de empresas, le permite a los empresarios tener mayor compromiso tanto con la empresa como con la sociedad (Cepinskis, Kvedaravicius, 2005), sin dejar de lado la responsabilidad que se debe tener en el manejo interno de sus propios recursos, ya sean materiales o humanos (Avram y Kühne, 2008). Respecto a los objetivos empresariales que tiene la PYME manufacturera, el factor de competitividad les demanda a los empresarios una importante atención, ya que dicho factor es vital para que su presencia en los mercados cuente con la credibilidad para cumplir con las exigencias de los clientes. Por lo anterior, es trascendente mencionar que la competitividad se define como la capacidad de generar una ventaja competitiva sostenible para producir bienes y servicios, los cuales generan un valor específico que permita la competencia con otras empresas (Hamel y Prahalad, 1989; Kay, 1993; Murths y Lenway, 1998; Porter y Kramer, 2006).

La competitividad en una organización se describe como la actividad que puede reflejar resultados diferenciados, tales como el funcionamiento del mercado, la capacidad de innovación y valores de los recursos procedentes de los recursos potenciales y diversos de la organización (Vogel, 2005; Álvarez, Marín y Fonfría, 2009; Intarapanich y Ussahawanitchakit, 2011). Para ello, los objetivos que se esperan en empresas altamente 
competitivas deben estar enfocados en tener un rendimiento superior, así como mantener la capacidad en el éxito de la operación (Ambastha y Momaya, 2004; Haigh y Jones, 2006; Porter y Kramer, 2006; Álvarez, Marín y Fonfría, 2009). Con base en el concepto de RS, las empresas tienen la responsabilidad de ofrecer productos de excelente calidad, pensar en la entera satisfacción de los clientes, lo que requiere la completa integración de empleados y del gobierno; en conjunto se pretende obtener un impacto sobre el medio ambiente y en la objetividad de los productos que se ofrecen al público en general (Jenkins, 2006, 2009; Lepoutre y Heene, 2006).

De acuerdo con Vogel (2005), la RS que generan las organizaciones (instituciones, organizaciones sin lucro, individuos, etc.) no es precisamente para sustituir las actividades que por obligación genera el gobierno para dar servicio y atención a la sociedad. Por el contrario, la RS que se genera en las organizaciones fortalece la relación entre el entorno social, a partir de la medición de sus necesidades primarias, el gobierno, a partir de su apertura y flexibilidad para aceptar el apoyo que generan socialmente las organizaciones no gubernamentales, y las propias organizaciones con la finalidad de coadyuvar en el crecimiento y desarrollo de los individuos, a partir del desempeño y competitividad de las mismas, para que, con todo ello, los ambientes tengan mejores índices de desarrollo y de seguridad social (Pradshaw y Vogel, 1980; Epsteinn, 2007).

Dentro del trabajo de investigación de Vogel (2005) resalta lo importante que es contar con políticas empresariales que favorezcan desde un punto de vista social a los trabajadores, con la finalidad de que las organizaciones, con este marco de estabilidad social, puedan tener mayor confiabilidad en el desarrollo organizacional y, en consecuencia, en la mejora del crecimiento del entorno. Finalmente, es relevante destacar la RS no como una estrategia genérica para cualquier negocio, sino que puede ser una op- 
ción para que determinadas organizaciones mejoren su desempeño y competitividad, según el entorno donde tengan su actividad operativa. Tal como lo señala Vogel (2005), la RS hace que los negocios tengan sentido para algunas empresas en algunas áreas bajo algunas circunstancias específicas.

La RS en su desarrollo dentro de la PYME manufacturera requiere, constantemente, no desviar su enfoque hacia el desempeño social (Swanson, 1995; Vogel, 2005), la ética en los negocios (Solomon, 1993), la relación con el gobierno corporativo (Freeman y Evans, 1990), el contrato social (Donaldson y Dunfee, 2002), la gestión con las secciones interesadas en alguna operación de negocios (Donaldson y Preston, 1995; Lozano, 2002) y la relación social con la ciudadanía (Waddock, 2000; Prahalad y Hammond, 2002). En este sentido, la RS se puede definir como la integración voluntaria de las preocupaciones sociales y empresariales en su interacción con las partes interesadas, donde parte de tales preocupaciones se apoyan en la visión y objetividad empresarial, en los códigos de ética empresariales, en los valores y en la reputación de la empresa (Pruzan, 2001; Whetten, Rands y Godfrey, 2001; Sum y Ngai, 2005). Bajo estas perspectivas se puede plantear la siguiente hipótesis:

$H_{1}:$ A mayor nivel de responsabilidad social corporativa, mayor nivel de competitividad de las PYMES manufactureras de Aguascalientes.

\subsection{Capital intelectual y la competitividad en la PYME manufacturera}

En años recientes la PYME manufacturera ha estado influenciada de manera significativa por los cambios que se han generado en la economía mundial y, en este sentido, los empresarios requieren estar más orientados en mejorar las estrategias de operación que hayan instrumentado, en donde 
aspectos como el capital intelectual, CI, en la actualidad debe estudiarse por parte de académicos y, además, es un foco de atención que debe ser de interés por parte de los empresarios de este tipo de organizaciones (Aragón y Rubio, 2002; Monagas, 2012). Asimismo, es importante mencionar que la PYME, en general, contribuye económicamente en las regiones con un porcentaje significativo, lo cual permite a estas empresas contar con una fuerza laboral estable y con oportunidades de crecimiento de gran dimensión (Carson, 1990; Saldivar et al., 2012).

En este sentido, es importante resaltar que el CI está definido por parte de los investigadores como una fuente de recursos, los cuales carecen de esencia tangible y pueden ser resultado de análisis financieros que, posteriormente, permita a las empresas ser más confiables y competitivas (Kaplan y Norton, 1992; Bontis, 1996; Edvinsson y Malone, 1999; Cañibano et al., 2002). Para ello, los empresarios deben tomar en cuenta que cualquier aportación por parte del CI en la PYME permitirá un crecimiento económico en el que se puedan lograr mayores niveles de competitividad (Vargas y Del Castillo, 2008). El CI es considerado como un aspecto de inversión que al aplicarse de manera activa en la economía de las empresas el rendimiento y competitividad empresarial será seguro de presentarse (Grant, 1996; Lev, 2001; González, Aguilar y Maldonado, 2011; Monagas, 2012).

El concepto de CI en la actualidad no es, desde luego, una nueva aportación por parte de los investigadores, sin embargo, para organismos autónomos como lo es la PYME, el tema de CI ha sido en los últimos años un elemento esencial de analizar y de integrar en el desarrollo de las organizaciones (Brooking, 1997; Edvinson y Malone, 1999). Algunos investigadores describen el CI como un material intelectual que puede utilizarse para crear un valor agregado a cualquier proceso interno de la empresa, el cual permita tener más riqueza con las aportaciones por parte de los em- 
pleados (Stewart, 1999). Por tal razón el CI es un elemento clave en la gestión del conocimiento (Rivero, 2009). Asimismo, el CI define a la posesión de conocimiento de los empleados y al conocimiento que se va generando en la sinergia natural que se da en la relación del trabajo, las actividades y las experiencias de los trabajadores (Stewart, 1999; Bontis, 2001).

Hay que resaltar que el CI es un recurso importante a tomar en cuenta, por parte de los empresarios, con el objeto de encontrar otras opciones de mejora que permitan crecer y eficientar las actividades operativas dentro de las organizaciones, al hacer uso de una mejor gestión empresarial (Vargas y del Castillo, 2008). De igual manera, el CI, al ser un elemento que integra habilidades y conocimientos, permitirá que las empresas como la PYME manufacturera sea más competitiva al aprovechar al máximo el conocimiento de todos sus empleados, en los niveles que la organización tenga activos (Fineman et al., 2005; Bogdam, Balint y Farcas, 2011; López-Gamero et al., 2011). Una empresa será competitiva siempre y cuando tenga el objetivo de cuidar el control de los costos, optimizar el uso de sus recursos y evaluar el desempeño de sus actividades operativas internas, para ello necesita que el CI sea aprovechado al máximo (Bogdam, Balint y Farcas, 2011). Bajo estas perspectivas se puede plantear la siguiente hipótesis:

$\mathrm{H}_{2}$ : A mayor nivel de capital intelectual, mayor nivel de competitividad de las PYMES manufactureras de Aguascalientes.

En la figura 1 se muestra el modelo teórico de la presente investigación y la representación de su ecuación teórica, mismo que da origen a la formulación de las hipótesis planteadas. 
Figura 1

Modelo teórico del constructo base de investigación

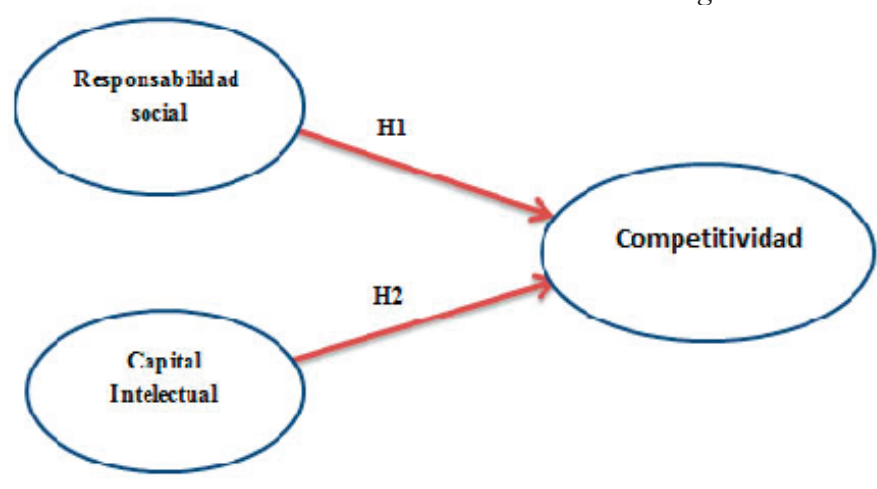

$$
\mathbf{Y}=\boldsymbol{\beta}_{0}+\boldsymbol{\beta}_{1}{ }^{*} \mathbf{x} 1+\boldsymbol{\beta}_{2}{ }^{*} \mathbf{x} 2+e
$$

La competitividad es $=\beta_{0}+\left(\beta_{1}{ }^{*}\right.$ responsabilidad social $)+\left(\beta_{2}{ }^{*}\right.$ capital in-

$$
\text { telectual) }+e
$$

\section{METODOLOGÍA}

\subsection{Diseño de la muestra y recolección de datos}

Se realizó una investigación empírica con un enfoque cuantitativo de tipo explicativo y transeccional. El instrumento base de investigación está compuesto por 55 ítems medidos en un escala tipo Likert de 1 a 5, donde refieren desde total desacuerdo hasta total acuerdo, el cual fue aplicado a los gerentes de las PYMES manufactureras del estado de Aguascalientes, México. Se analizó la responsabilidad social y el capital intelectual en la PYME manufacturera de Aguascalientes para una mejor competitividad empresarial. Para el desarrollo de la investigación se tomó de referencia la base de datos que ofrece el Directorio empresarial 2014 del sistema de información empresarial de México (SIEM) del estado de Aguascalientes, en el aparecen registradas hasta el 14 de enero de ese mismo año, 5209 
empresas, de las cuales 793 pertenecen al sector industrial y, de éstas, únicamente 250 son PYMES de 11 a 250 trabajadores. Por lo que se obtuvo una muestra de 150 PYMES del sector industrial manufacturero en Aguascalientes, a cuyos gerentes se les aplicó el instrumento de medición tipo encuesta personalizada de manera aleatoria simple, con un nivel de confianza de $95 \%$ y un margen de error de 5.1 por ciento. Dicha información se puede apreciar en el cuadro 1, mismo que hace referencia al diseño de la investigación.

\section{Cuadro 1}

Diseño de la investigación

\begin{tabular}{|ll|}
\hline \multicolumn{1}{|c|}{ Características } & \multicolumn{1}{c|}{ Investigación } \\
Población* & 250 Pequeñas y medianas empresas \\
Área geográfica & Estado de Aguascalientes, México \\
Objeto de estudio & Pymes manufactureras de 11 a 250 trabajadores \\
Sector industrial al que pertenecen & Metalmecánica, textil, agroindustrial y mueblero \\
\cline { 2 - 2 } Método de recolección de información & Entrevistas personales a los gerentes \\
Método de muestreo & Muestreo aleatorio simple \\
Tamaño de la muestra & 150 Pymes \\
Error del muestreo & $\pm 5.1 \%$ error, nivel de confiabilidad del $95 \%(\mathrm{p}=\mathrm{q}=0$ \\
\hline Trabajo de campo & Septiembre a Octubre de 2012 \\
\hline
\end{tabular}

Fuente: Sistema de información empresarial de México (SIEM), 2014.

Antes de realizar el cuestionario se hizo una prueba piloto del mismo con un grupo de expertos, para asegurarse que las preguntas son fácilmente comprensibles y así evitar que el encuestado generara errores por una interpretación incorrecta de la pregunta. De igual manera, el instrumento se sometió a una prueba de fiabilidad estadística, ésta fue llevada a cabo a través del coeficiente alpha de Cronbach del constructo base del instrumento, cuyos resultados arrojados por dicha prueba fueron de .962, 
con lo que se puede interpretar que el estudio es fiable y valido (Nunnally y Bernstein, 1994). Para la preparación del instrumento de medición, se utilizaron 3 bloques los cuales se describen a continuación, y cuyos resultados de fiabilidad se muestran en el apéndice 1.

Bloque I. Competitividad. Compuesto por 18 ítems que en su totalidad cuentan con un alpha de Cronbach de .922; en el que se consideraron tres factores elementales: desempeño financiero, compuesto por 6 ítems, con un alpha de Cronbach de .917; reducción de los costos de las compras, 6 ítems, con un alpha de Cronbach de .808 y uso de tecnología, 6 ítems, con un alpha de Cronbach de .932; adaptada de Buckley, Pass y Prescott (1988) y Chang, et al. (2005); por lo tanto los tres constructos que componen el bloque de competitividad se pueden interpretar como fiables y validos (Nunnally y Bernstein, 1994).

Bloque II. De capital intelectual. Compuesto por 18 ítems, en su totalidad cuentan con un alpha de Cronbach de .916; en el se tomaron en cuenta tres factores elementales: búsqueda de la información, compuesto por 5 ítems, con un alpha de Cronbach de .792; desarrollo del conocimiento, 5 ítems, con un alpha de Cronbach de .881 y aprendizaje y retroalimentación, 8 ítems, con un alpha de Cronbach de .877; tomada de Cañibano, et al. (2002), así los tres constructos del bloque de capital intelectual se pueden interpretar como fiables y validos (Nunnally y Bernstein, 1994).

Bloque III. De responsabilidad social. Compuesto por 19 ítems, en su totalidad cuentan con un alpha de Cronbach de .924; en el se consideraron cuatro factores elementales: instrumentación de programas, compuesto por 4 ítems, con un alpha de Cronbach de .940; acciones de la responsabilidad social, 7 ítems, con un alpha de Cronbach de .915; involucramiento, 4 ítems, con un alpha de Cronbach de .923 y costos de instrumentación, 4 ítems, con un alpha de Cronbach de .868; tomado de Elías (2004), por 
ello los cuatro constructos que forman el bloque de responsabilidad social pueden considerarse fiables y validos (Nunnally y Bernstein, 1994).

En el estudio se aplicó un análisis multivariante de datos, se utilizó como técnica estadística el análisis de regresión lineal múltiple, a través del método de pasos sucesivos, a fin de identificar la relación que existe entre las variables explicativas capital intelectual y responsabilidad social con la competitividad, mediante los valores de los coeficientes de beta, y de igual manera identificar el valor de $\mathrm{R}^{2}$; para de esta forma conocer qué tanto las variables materia de estudio explican la competitividad. Es de destacar que, para llevar a cabo la regresión lineal múltiple, fue necesario generar una nueva variable por cada una de las variables explicativas y la dependiente, con la media de todos los ítems que componen a éstas. Posteriormente, se introdujeron ambas variables explicativas (responsabilidad social y capital intelectual) como variables independientes y la variable competitividad como variable dependiente, en el software estadístico IBM, SPSS Statistics V21, aplicando la regresión lineal múltiple bajo el método de pasos sucesivos, se utilizó el criterio de los valores de $F$ para entrar $\geq 3.840$ y $F$ para salir $\leq 2.710$.

De igual manera se llevó a cabo un análisis correlacional de las variables explicativas con la variable dependiente, a fin de conocer la correlación que existe entre éstas, tal y como se muestra en los resultados.

\section{RESULTADOS}

Primero se realizó el análisis de correlación de pearson, cuyos resultados, que se muestran en el cuadro 2 , indican que la responsabilidad social y la competitividad están correlacionadas en $70.8 \%$ y que es significativa a un 
valor de $\mathrm{p}<0.001$. Asimismo, el capital intelectual está correlacionado con la competitividad en $65.8 \%$ y es significativa a un valor de $\mathrm{p}<0.001$, la variable responsabilidad social y el capital intelectual están correlacionadas entre sí en $60.9 \%$ y es significativa a un valor de $\mathrm{p}<0.001$.

\section{Cuadro 2}

Correlaciones

\begin{tabular}{|c|c|c|c|c|}
\hline & & Competitividad & $\begin{array}{c}\text { Responsabilidad } \\
\text { social }\end{array}$ & $\begin{array}{r}\text { Capital } \\
\text { intelectual }\end{array}$ \\
\hline \multirow{3}{*}{ COMPETITIVIDAD } & Correlación de Pearson & 1 & .708 & .658 \\
\hline & Sig. (bilateral) & & .000 & .000 \\
\hline & $\mathrm{N}$ & 150 & 150 & 150 \\
\hline \multirow{3}{*}{$\begin{array}{c}\text { RESPONSABILIDAD } \\
\text { SOCIAL }\end{array}$} & Correlación de Pearson & .708 & 1 & .609 \\
\hline & Sig. (bilateral) & .000 & & .000 \\
\hline & N & 150 & 150 & 150 \\
\hline \multirow{3}{*}{$\begin{array}{c}\text { CAPITAL } \\
\text { INTELECTUAL }\end{array}$} & & .658 & & 1 \\
\hline & Sig. (bilateral) & .000 & .000 & \\
\hline & $\mathrm{N}$ & 150 & 150 & 150 \\
\hline
\end{tabular}

Posteriormente, con el propósito de verificar las condiciones de aplicabilidad del análisis de regresión lineal múltiple aplicado al modelo de investigación para determinar el impacto de la responsabilidad social y el capital intelectual en la competitividad de las PYMES manufactureras de Aguascalientes, se llevaron a cabo las pruebas de normalidad, homoscedasticidad y linealidad; encontrándose que las variables objeto de estudio no presentan problema en ninguna de las tres, por lo que se procedió al análisis de la regresión lineal múltiple a través del software estadístico IBM, SPSS Statistics V21, en el cuadro 3 se presenta el resumen del modelo, en el se obtuvo un valor de $\mathrm{R}$ de .764 y una $\mathrm{R}^{2}$ de .584 , lo que indica que juntas las variables responsabilidad social y capital intelectual están correlacionadas en 76.4\% con la competitividad de las PYMES manufactureras de Aguascalientes y en conjunto explican en $57.8 \%$ la competitividad de las PYMES en cuestión. 


\section{Cuadro 3}

Resumen del modelo de la muestra total ${ }^{c}$

\begin{tabular}{|c|c|c|c|c|c|}
\hline Modelo & $R$ & $R$ cuadrado & corregida & $\begin{array}{c}\text { Error típ. de la } \\
\text { estimación }\end{array}$ & $\begin{array}{l}\text { Durbin- } \\
\text { Watson }\end{array}$ \\
\hline & .708 & .502 & .498 & .567 & \\
2 & .764 & .584 & .578 & .520 & 1.223 \\
\hline
\end{tabular}

Fuente: Elaboración propia con base en resultados de regresión lineal múltiple.

De acuerdo con los resultados de la regresión lineal múltiple presentados en el cuadro 4 se concluye que: 1) alrededor de $48.8 \%$ de la competitividad de las PYMES manufactureras de Aguascalientes se debe a la responsabilidad social, al influir ésta de manera positiva y significativa en la competitividad, con un valor de $\mathbf{t}$ de 7.281 , a un nivel de significancia de 0.001 ; 2) la variable capital intelectual de igual forma influye de manera positiva y significativa en $36.1 \%$ en la competitividad de las PYMES manufactureras de Aguascalientes, con un valor de $\mathbf{t}$ de 5.381, a un nivel de significancia de 0.001 y 3) juntas las variables responsabilidad social y capital intelectual explican en 57.8\% la competitividad, con un valor de F de 103.046, el cual es significativo por ser su valor de $p<0.001$. En cuanto a los estadísticos de colinealidad se obtuvo un FIV de 1.589, lo que indica que el modelo se encuentra en el límite de la multicolinealidad. 


\section{Cuadro 4}

Resultados análisis de regresión lineal múltiple

\begin{tabular}{|c||c|}
\hline Variables & Competitividad \\
\hline Responsabilidad social & $0.488^{* * *}$ \\
$(7.281)$ \\
Capital intelectual & $0.361^{* * *}$ \\
$\mathrm{R}^{2}$ ajustada & $(5.381)$ \\
\hline Valor de $\mathrm{F}$ & 0.578 \\
FIV más alto & $103.046^{* * *}$ \\
\hline
\end{tabular}

Fuente: Elaboración propia con base en resultados de regresión lineal múltiple.

Asimismo, el modelo ha sido validado al dividirse la muestra en dos submuestras y al haberlo corrido con las dos submuestras, los resultados obtenidos son similares en cuanto al $\mathrm{R}^{2}$, por no haber más de $10 \%$ de diferencia entre éstos y la muestra original (Hair et al., 1998), la submuestra 1 , con 74 observaciones de las 150 de la muestra original se obtuvo un valor de $\mathrm{R}^{2}$ ajustada de .510 y la submuestra 2 que contempla las 76 observaciones restantes de las 150 , se obtuvo un valor de $\mathrm{R}^{2}$ ajustada de .637 , por lo tanto, no existe más de $10 \%$ de diferencia con el valor de la muestra total original, tal y como se muestra en el cuadro 5 . 
Cuadro 5

Resumen del modelo de las dos submuestras ${ }^{c}$

\begin{tabular}{|c|c|c|c|c|c|c|}
\hline Submuestra 1 con & Modelo & $\mathrm{R}$ & $\mathrm{R}$ cuadrados & $\begin{array}{c}\text { R cuadrados } \\
\text { corregidos }\end{array}$ & $\begin{array}{c}\text { Error tip. de la } \\
\text { estimación }\end{array}$ & $\begin{array}{c}\text { Dubin } \\
\text { Watson }\end{array}$ \\
\hline 1 & $646 \mathrm{a}$ & 417 & 409 & 583 & 1545 \\
\hline
\end{tabular}

a. variables predictoras (constante). CAPITALINTELECTUAL

b. Variables predictoras (constante). CAPITALINTELECTUAL. RESPONSABILIDADSOCIAL

c. Variable dependiente. COMPETITIBIDAD

\begin{tabular}{|c|c|c|c|c|c|c|}
\hline & \multicolumn{6}{|c|}{ Resumen del modelo ${ }^{\text {a }}$} \\
\hline & Modelo & $\mathrm{R}$ & $\mathrm{R}$ cuadrados & $\begin{array}{c}\mathrm{R} \text { cuadrados } \\
\text { corregidos }\end{array}$ & $\begin{array}{c}\text { Error tip. de la } \\
\text { estimación }\end{array}$ & $\begin{array}{l}\text { Dubin } \\
\text { Watson }\end{array}$ \\
\hline 76 observaciones & $\begin{array}{l}1 \\
2\end{array}$ & $\begin{array}{l}.779 \mathrm{a} \\
.804 \mathrm{~b}\end{array}$ & $\begin{array}{l}.607 \\
.647\end{array}$ & $\begin{array}{l}.601 \\
.637\end{array}$ & $\begin{array}{l}529 \\
504\end{array}$ & 1216 \\
\hline
\end{tabular}

a. variables predictoras (constante). RESPONSABILIDADSOCIAL

b. Variables predictoras (constante). RESPONSABILIDADSOCIAL. CAPITALINTELECTUAL

c. Variable dependiente. COMPETITIBIDAD

Fuente: Elaboración propia con base en resultados de regresión lineal múltiple.

Ahora bien, con base en los resultados contenidos en el cuadro 6 se procedió a identificar los coeficientes no estandarizados de beta para cada una de las variables, que en este caso son:

$$
\beta_{0}=.492 ; \beta_{1}=.463 ; y \beta_{2}=.380
$$

\section{Cuadro 6}

Coeficientes $^{a}$

\begin{tabular}{|c|c|c|c|c|c|c|c|c|c|c|c|}
\hline & & \multicolumn{2}{|c|}{$\begin{array}{l}\text { Coeficientes no } \\
\text { estandarizados }\end{array}$} & \multirow{2}{*}{$\begin{array}{c}\begin{array}{c}\text { Coeficientes } \\
\text { tipificados }\end{array} \\
\text { Beta } \\
\end{array}$} & \multirow[b]{2}{*}{$\mathrm{t}$} & \multirow[b]{2}{*}{ Sig. } & \multicolumn{3}{|c|}{ Correlaciones } & \multicolumn{2}{|c|}{$\begin{array}{c}\text { Estadísticos de } \\
\text { colinealidad }\end{array}$} \\
\hline \multicolumn{2}{|c|}{ Modelo } & B & Error típ. & & & & Orden cero & Parcial & Semiparcial & Tolerancia & FIV \\
\hline & (Constante) & 1.220 & .172 & & 7.100 & .000 & & & & & \\
\hline \multirow{2}{*}{2} & (Constante) & .492 & .208 & & 2.366 & .019 & & & & & \\
\hline & CAPITALINTELECTUAL & .380 & .071 & .361 & 5.381 & .000 & .658 & .406 & .286 & .629 & 1.589 \\
\hline
\end{tabular}

Fuente: Elaboración propia con base en resultados de regresión lineal múltiple. 
De igual manera se identificaron los valores de las medias arrojados en los estadísticos descriptivos de los resultados de la regresión lineal múltiple, éstos fueron de 3.0102 para la responsabilidad social y de 3.5708 para el capital intelectual, por lo que, de acuerdo con lo anterior, a continuación se presenta el valor de $\mathbf{Y}$, que representa la competitividad de las PYMES manufactureras de Aguascalientes, lo que nos indica que, con base en la ecuación de regresión, la competitividad está en función de las variables responsabilidad social y capital intelectual en una media de 3.24, con un máximo de 6.45 y un mínimo de 0.033 , al utilizar dos errores estándar.

Competitividad $=\beta_{0}+\left(\beta_{1}{ }^{*}\right.$ responsabilidad social $)+\left(\beta_{2}{ }^{*}\right.$ capital intelectual $)+e$

Competitividad $=0.492+(0.463 * 3.0102)+(0.38 * 3.5708)+1.6047=3.2426$

A fin de analizar la presencia de observaciones anómalas, de acuerdo con la gráfica 1, se muestra que sí hay algunos datos que fungen como outliers, los cuales se presentan en la parte superior de la gráfica, por encima de la línea roja, e inferior, por debajo de la línea roja, que marca el límite de los dos errores estándar.

Gráfica 1

Residuos estudentizados

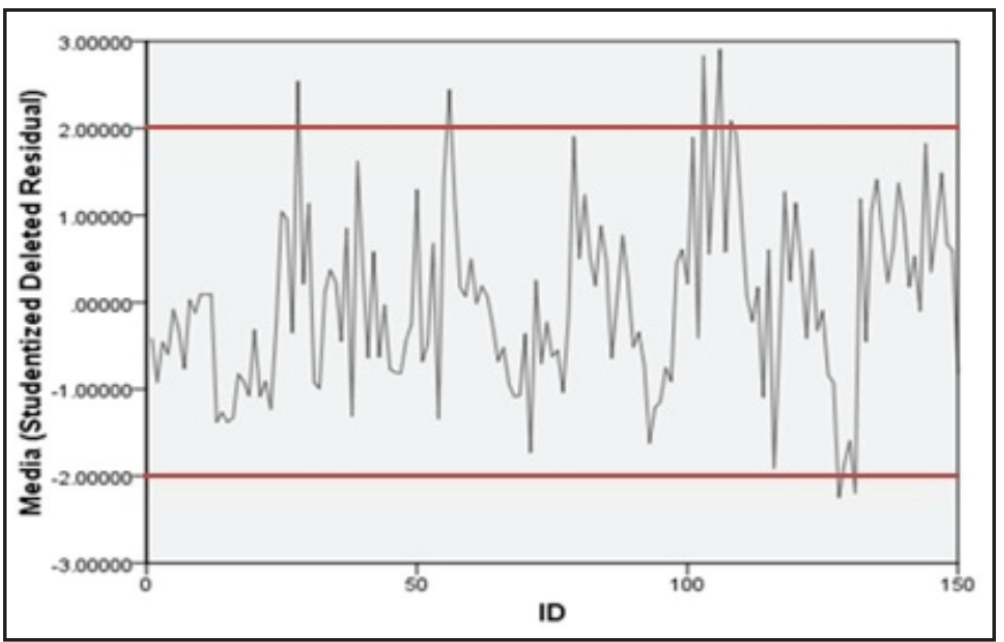

Fuente: Elaboración propia con base en los resultados de la regresión lineal múltiple. 
Por lo tanto, respecto a las hipótesis formuladas en la presente investigación se procede a su comprobación, en relación con la $\mathrm{H}_{1}$, los resultados obtenidos $(\beta=0.488, \mathrm{p}<0.001)$ indican que la responsabilidad social tiene efectos significativos en la competitividad de las PYMES manufactureras de Aguascalientes. En virtud de que la responsabilidad social influye positivamente en $48.8 \%$ en la competitividad de dichas PYMES, se acepta la $\mathrm{H}_{1}$. En cuanto a la $\mathrm{H}_{2}$, los resultados obtenidos $(\beta=0.361$, $\mathrm{p}$ $<0.001)$ muestran que el capital intelectual tiene efectos significativos en la competitividad de las PYMES manufactureras de Aguascalientes. En virtud de que el capital intelectual influye positivamente en $36.1 \%$ en la competitividad de las PYMES en cuestión, se acepta la $\mathrm{H}_{2}$.

En lo que respecta al objetivo de la presente investigación, se concluye que tanto la responsabilidad social como el capital intelectual influyen de manera significativa en la competitividad de las PYMES manufactureras del estado de Aguascalientes, por lo que es esencial para las empresas el poner especial cuidado en estas dos variables, ya que pueden tener una influencia positiva a largo plazo en el logro de las metas y objetivos de las organizaciones y, con ello, alcanzar el éxito competitivo en un mercado global.

\section{DISCUSIÓN}

Los resultados obtenidos en este trabajo demuestran que la responsabilidad social y el capital intelectual tienen implicaciones importantes en la competitividad de las PYMES manufactureras de Aguascalientes. Por esta razón, la responsabilidad social representa un potencial que puede tener una influencia positiva a largo plazo en el logro de las metas y obje- 
tivos de las organizaciones. Por lo tanto, los resultados obtenidos pueden ser interpretados como requisitos esenciales para determinar el nivel de competitividad de las PYMES. Asimismo, el capital intelectual representa una diferencia sustancial que puede facilitar el logro de las metas de las organizaciones.

Al analizar los trabajos más recientes sobre el tema, se observa que la pequeña y mediana empresa está interesada por la responsabilidad social, ya que se pueden atender con facilidad aspectos sociales, laborales y ambientales con un enfoque específico de mejorar el bienestar de los trabajadores (Avram y Kühne, 2008), también se puede observar que la PYME, en especial la manufacturera, tiene algunas limitaciones que, en ocasiones, detienen el desarrollo y crecimiento de las organizaciones. En el tema empresarial, la gestión de los recursos financieros, el control de los apoyos externos o el adecuado manejo de las habilidades de los empresarios y líderes deben enfocarse a que la sociedad en general considere a este tipo de organizaciones comprometidas con la responsabilidad social (Cepinskis, Kvedaravicius y Zirgulis, 2005; Jenkins, 2009; Laurinkevidiciute y Stasiskiene, 2010). Al integrarse la responsabilidad social a la actividad laboral de estas empresas le permite a los empresarios tener más compromiso tanto con la empresa como con la sociedad (Cepinskis, Kvedaravicius y Zirgulis, 2005), sin dejar de lado la responsabilidad que se debe tener con el manejo interno de sus propios recursos, ya sean materiales o humanos (Avram y Kühne, 2008).

En lo que se refiere al capital intelectual es importante resaltar que es una fuente de recursos que carecen de esencia tangible y que pueden ser resultados de análisis financieros que, posteriormente, permita a las empresas ser más confiables y competitivas (Kaplan y Norton, 1992; Bontis, 1996; Edvinsson y Malone, 1997; Cañibano et al., 2002). Para ello, 
los empresarios deben tomar en cuenta que cualquier aportación por parte del capital intelectual en la PYME permitirá un crecimiento económico en donde se puedan lograr mayores niveles de competitividad (Vargas y del Castillo, 2008).

\section{CONCLUSIONES}

En la actualidad para los gerentes de las PYMES manufactureras de Aguascalientes, la responsabilidad social es un tema que ha permitido a los empresarios poner una especial atención en evaluar los beneficios que se tendrán, en muchos sentidos, en sus organizaciones, sobre todo, en lo referente al desarrollo económico social; lo que indica que las empresas le han apostado más al desarrollo económico social a fin de que se vea reflejado en sus actividades de responsabilidad social corporativa. En cuanto a la dimensión de instrumentación, la variable que más aporta a la RS es la (RSG4), relativa a que las acciones de responsabilidad social corporativa son tomadas como una actividad más a realizar en la empresa, equiparada en importancia a las demás, lo que se ha visto reflejado en las actividades de RS que realizan para alcanzar el éxito competitivo. Sobre la dimensión de involucramiento la variable que más aporta, de acuerdo con las interpretaciones de los gerentes, es la (RSI4) relativa a que las PYMES consideran que los empleados tienen conocimiento del programa de responsabilidad social corporativa y participan activamente en él. De la dimensión de costos de instrumentación, los gerentes consideran que el establecimiento de un programa de RSC representa una inversión que no retorna beneficio económico para su empresa (RSB2).

Acerca de las variables de la RS que más influyen en la competitividad de las PYMES, con base en la interpretación de los gerentes, son 
la (RSP1) filantropía, la cual está correlacionada de manera directa con la competitividad en $30.8 \%$; la (RSP2), que se refiere a las contribuciones que puede otorgar la empresa desde un punto de vista social, humano y empresarial, está correlacionada con la competitividad en 34.5\%; ambas variables a un nivel de significancia de $\mathrm{p}<0.001$, por último, la otra variable que está correlacionada con la competitividad, en $9.9 \%$, es la (RSB3) relativa a que el costo de tener en funcionamiento un programa de RSC es mayor que el beneficio recibido, la cual es significativa con un valor de $\mathrm{p}<0.05$.

Por lo anterior, se puede concluir que las PYMES que realizan actividades de responsabilidad social corporativa (RSC) son más propensas a lograr el éxito competitivo, en comparación con aquellas que no realizan actividad de ningún tipo, como la filantropía o las contribuciones que tiene la empresa con su personal, ya sea desde un punto de vista empresarial, de crecimiento o del desarrollo de cada empleado y, desde luego, con su incidencia en la sociedad en general. Sin embargo, nos pudimos percatar de que algunos gerentes de las PYMES consideran que el tener en funcionamiento un programa de RSC les es más costoso, en comparación con el beneficio que ellos tienen, ya que éste no se ve reflejado de manera inmediata en su organización.

En cuanto al capital intelectual, de la dimensión de búsqueda de la información la variable que más aporta al CI es la (CIB3), relativa a la información proveniente de tecnologías como internet, bases de datos, etc.; de la dimensión de desarrollo de conocimiento, la variable que más contribuye al CI es la (CIC3), que anticipa potenciales oportunidades de mercado para nuevos productos/servicios y de la dimensión de aprendizaje y retroalimentación, la variable que más participa en el CI es la (CIA6), en la que la PYME liga fuentes de conocimiento a problemas y retos.

De acuerdo con las interpretaciones de los gerentes, las variables del capital intelectual que más influyen en la competitividad de las 
PYMES son capaces de localizar y aplicar conocimientos para condiciones cambiantes de competitividad (CIA8), toda vez que dicha variable está correlacionada de manera directa con la competitividad en $21.4 \%$ y que las PYMES identifican nuevas oportunidades de negocio (CIC1), correlacionada con la competitividad en $20.6 \%$, ambas son significativas con un valor de $\mathrm{p}<0.001$.

Con base en lo anterior, se puede deducir que las PYMES que están realizando actividades de capital intelectual son más competitivas que las que no las han realizado, como son el tener la capacidad de localizar y aplicar conocimientos para actividades cambiantes de competitividad y el identificar nuevas oportunidades de negocio, como parte de sus actividades de capital intelectual.

Por lo tanto, los resultados obtenidos en el presente estudio son de gran valor, tanto para los gerentes de las PYMES mexicanas como para los diseñadores de las políticas públicas, ya que los gerentes podrán darse cuenta de cómo está influyendo la responsabilidad social para una mayor competitividad, así como de la influencia que tiene el capital intelectual dentro de la organización. Esto a través de la búsqueda de la información, el desarrollo del conocimiento y del aprendizaje y retroalimentación y, en consecuencia, de cómo se puede ver reflejado en su rendimiento financiero, al realizar algún tipo de actividad de responsabilidad social corporativa o de capital intelectual, para de esta manera tomar las mejores decisiones al momento de invertir.

La investigación presenta algunas limitaciones que sugieren vías de investigación futuras. El estudio es de corte transversal y estudios posteriores longitudinales podrían verificar el alcance de la responsabilidad social y el capital intelectual en la competitividad más a largo plazo. De igual manera, se sugiere trabajar con una muestra mayor de empresas, ya que esta investigación sólo consideró una muestra de 150 PYMES. Fi- 
nalmente, incluir factores de control como la edad de la empresa, la estructura familiar de la propiedad y las características profesionales de los gerentes, podrían ayudar a explicar diferencias de comportamiento en la relación responsabilidad social y capital intelectual en la competitividad de las PYMES manufactureras de Aguascalientes.

\section{REFERENCIAS}

Álvarez, I., R. Marín y A. Fonfría. 2009. The role of networking in the competitiveness of firms, Technological Forecasting \& Social Change, 76: 410-421.

Ambastha, A. y K. Momaya. 2004. Competitiveness of firms: review of theory, frameworks and models, Singapore Management Review, 26(1): 45-61.

Aragón, S. A. y B.A. Rubio. 2002. Factores explicativos del éxito competitivo. Un estudio empírico de la Pyme, Cuadernos de Gestión, 2(1): 49-63.

Avram, D.O. y S. Kühne. 2008. Implementing responsible business behavior from a strategic management perspective: developing a framework for Austrian SMEs, Journal of Business Ethics, 82: 463-475.

Bagdoniené, D. y E. Paulavicienè. 2010. Socialinès atsakomybés ir organizaeijos vadybos sistemos integravimas (The integration of social responsibility and organization's management system). Ekonomika ir Vadyba (Economies and Management), 15: 366-373.

Beyers, W.B. 2006. On the regional decline in manufacturing employment in the United States, documento presentado en 53rd Annual North American Meeting of the Regional Science Association, Toronto, noviembre 16-18. 
Bibri, M. 2008. Corporate sustainability/CSR communications \& value creation: A marketing approach, School of Management Blekinge, Institute of Technology, tesis de maestría (MBA).

Bodgdam, V., J. Balint y M. Farcas. 2011. Intellectual capital reporting and disclosure in the annual reports of Romanian manufacturing listed companies-theoretical framework, Annals of the University of Oradea, Economic Science Series, 1(1): 275-283.

Bontis, N. 1996. Intellectual capital: An exploratory analysis that develops measures and models, Management Decision, 36(4): 63-76.

2001. Assessing knowledge assets: A review of the models used to measure intellectual capital, International Journal of Management Reviews, 3(1): 41-60.

Bounfour, A. 2003. The management of intangibles: The organization's most valuable assets, London: Routledge.

Brooking, A. 1997. El capital intelectual. Barcelona, Paidós, www.gestiondelconocimiento.com/ biblio grafia_conceptos.htm

Bryson, J.R., M. Taylor y R. Cooper. 2008. Competing by design, specialization and customization: Manufacturing locks in the west midlands (UK), Geografiska Annaler:Series B, Human Geography, 90(2): 173-186.

Buckley, J.P., L.C. Pass y K. Prescott. 1988. Measures of international competitiveness: A critical survey, Journal of Marketing Management, 4(2): 175-200.

Cañibano, L., M. García-Ayuso, P. Sánchez y C. Chaminade. 2002. Directrices para la gestión y difusión de información sobre intangibles: informe de capital intelectual, Fundación Airtel Móvil, Madrid.

Carson, D. 2000. Marketing at the interface: not "what" but "how", Journal of Marketing Theory and Practice, 8: 18-19. 
Cepinskis, J., J. Kvedaravicius y V. Zirgutis. 2005. Development of small and medium sized enterprises in environmental aspect, Environmental Research, Engineering and Management, 4(34): 89-96.

Chang, S.C., R.J. Lin, J.H. Chen y L.H. Huang. 2005. Manufacturing flexibility and manufacturing proactiveness: Empirical evidence from the motherboard industry, Industrial Management \& Data System, 105(8): 1115-1132.

Donaldson, T. y L.E. Preston. 1995. The stakeholder theory of the corporation: Concepts, evidence, and implications, Academy of Management Review, 20: 65-91.

Donaldson, T. y T.W. Dunfee. 2002. Ties that bind in business ethics: Social contracts and why they matter, Journal of Banking \& Finance, 26(9): 1853-1865.

Edvinson, L. y M. Malone. 1999. El capital intelectual: cómo identificar y calcular el valor de los recursos intangibles de su empresa, Barcelona, Gestión 2000.

Elias, R.Z. 2004. An examination of business students' perception of corporate social responsibilities before and after bankruptcies, Journal of Business Ethics, 52: 267-281.

Epsteinn, E.M. 2007. The good company: Rhetoric or reality? Corporate social responsibility and business ethics redux, American Business Law Journal, 44 (2): 207-222.

Fineman, I., C. Giza, B. Nahed, S.M. Lee y D. Hovda. 2005. Inhibitions of neocordical plasticity during development by a moderate consecutive brain injury, Journal of Neotrauma, 17(1): 739-749.

Freeman, R. E. y W.M. Evan. 1990. Corporate governance: A stakeholder interpretation, Journal of Behavioral Economics, 19(4): 337-359.

González, A.M., R.H. Aguilar y G.G. Maldonado. 2011. Capital intelectual: innovación indicador del capital estructural en empresas del 
sector manufacturero en Aguascalientes, Global Conference on Business and Finance Proceedings, 6(2): 1080-1088.

Grant, R.M. 1996. Prospering in dynamically-competitive environments: Organizational capability as knowledge integration, Organization Science, 7(4): 375-378.

Haigh, M. y M.T. Jones. 2006. The divers of corporate social Responsibility: A critical review, The Business Review, Cambridge, 5(2): 245-251.

Hair, J.F., R.E. Anderson, R.L. Tatham y W.C. Black. 1998. Multivariate Data Analysis ( $5^{\text {th }}$ Edition). New Jersey, Prentice Hall.

Hamel, G. y C.K. Prahalad. 1989. Strategic intent, Harvard Business Review, 3: 63-76.

Intarapanich, S. y P. Ussahawanitchakit. 2011. Dynamic technology capability, firm competitiveness enhancement, and organizational stability: Evidence from IT businesses in Thailand, Journal of International Business and Economics, 11(4): 93-120.

Jenkins, H. 2006. Small business champions for corporate social responsibility, Journal of Business Ethics, 67(3): 241-256.

2009. A business opportunity model of corporate social responsibility for small- and medium-sized enterprises, Business Ethics: A European Review, 18(1): 21-36.

Kaplan, R.S. y D.P. Norton. 1992. The balanced scorecard-measures that drive performance, Harvard Business Review, 1(1): 71-79.

Kay, J. 1993. Foundations of Corporate Success, Oxford, Oxford University Press.

Laurinkeviciute, A. y Z. Stasiskiene. 2010. Sustainable development decision-making model for small and medium enterprises. Environmental research, Engineering and Management, 2(52): 14-24. 
Lepoutre, J. y A. Heene. 2006. Investigating the impact of firm size on small business social responsibility: A critical review, Journal of Business Ethics, 67(3): 257-273.

Lev, B. 2001. Intangibles: Management, Measurement and Reporting, Washington, DC, The Brookings Institute.

Lipovatz, D., M. Mandaraka y A. Mourelatos. 2000. Multivariate analysis for the assessment of factors affecting industrial competitiveness: The case of Greek food and beverage industries, Applied Stochastic Models in Business and Industry, 16(2): 85-98.

López-Gamero, M., P. Zaragoza-Saenz, E. Claver-Cortés y J. Molina-Azorin. 2011. Sustainable development and intangibles: Building sustainable intellectual capital, Business Strategy \& the Environment, 20(1): 18-37.

Lozano, J.M. 2002. Towards the relational corporation: From managing stakeholder relations to building stakeholder relationships (waiting for Copernicus), Corporate Governance, 5(2): 60-77.

Monagas, D.M. 2012. El capital intelectual y la gestión del conocimiento, Ingeniería Industrial, 33(2): 142-150.

Murths, T. P. y S.A. Lenway. 1998. Country capabilities and the strategic state: How national political institutions affect MNC strategies, Strategic Management Journal, 15(5): 113-119.

Nunnally, J.C. e I.H. Bernstein. 1994. Psychometric Theory, 3a. ed. New York, McGrawHill.

Porter, M.E. y M.R. Kramer. 2006. Strategy and society: The link between competitive advantage and corporate social responsibility, Harvard Business Review, 84(12): 78-92.

Pradshaw, T. y D. Vogel. 1980. Corporations and their Critics:Issues and Answers on the Problems of Corporate Social Responsibility, NY, McGraw-Hill Book Company. 
Prahalad, C. K. y A. Hammond. 2002. Serving the world's poor, profitably, Harvard Business Review, 80(9): 48-57.

Pruzan, P. 2001. The question of organizational consciousness: Can organizations have values, virtues and visions? Journal of Business Ethics, 29(3): 271-284.

Rivero, D. 2009. Modelo conceptual para la medición del capital intelectual y un procedimiento para su implementación. Caso hotelero, tesis doctoral, Cuba, Universidad de Matanzas.

Roos, G., S. Pike y L. Fernström. 2005. Managing Intellectual Capital in Practice, Oxford, Butterworth-Heinemann.

Saldivar, G.S.J., R.B.R. García, M.N. Valenciana y R.R.O. Roa. 2012. Competitividad y gestión de las PYMES, Global Conference on Business and Finance proceedings, 7(2): 1152-1156.

Sistema de información Empresarial Mexicano. Obtenido de Directorio de Empresas: http://www.siem.gob.mx/siem/estadisticas/estadotamanoPublico.asp?tam $=3 \& p=1$

Smith, N.C. 2003. Corporate social responsibility: Whether or how? California Management Review, 45(4): 52-76.

Solomon, R.C. 1993. Ethics and Excellence. Cooperation and Integrity in Business, Oxford, Oxford University Press.

Stewart, T. 1997. Intellectual Capital: The New Wealth of Organisations, NY, Doubleday.

Sum, N. y P. Ngai. 2005. Globalization and paradoxes of ethical transnational production: Code of conduct in Chinese workplace, Competition \& Change, 9(2): 181-200.

Swanson, D.L. 1995. Addressing a theoretical problem by reorienting the corporate social performance model, Academy of Management Review, 20: 43-64. 
Szymura-Tyc, M. 2009. The role of marketing intellectual capital in creating competitive advantage in the international market-theoretical assumptions and empirical evidence of polish firms competing in the European markets, Journal of Economics \& Management, 6(1): 161-186.

Ussahawanitchakit, P. 2005. Effect of E-commerce on export marketing strategy and performance: An empirical study of Thai firms, Review of Business Research, 5(3): 46-54.

Van de Ven, B. y R. Jeurissen. 2005. Competing responsibly, Business Ethics Quarterly, 15(2): 299-317.

Vargas, B. y C. del Castillo. 2008. Competitividad sostenible de la pequeña empresa: un modelo de promoción de capacidades endógenas para promover ventajas competitivas sostenibles y alta productividad, Cuadernos de Difusion, 13(24), 59-80.

Vázquez, A.G., G.J. Sánchez y C.R. Rodríguez. 2012. Impact of knowledge management and intellectual capital on competitiveness of SME's manufacturing in the Western region of Mexico, Competitive Forum, 10(1): 56-62.

Vogel, D. 2005. The Market for Virtue: The Potential and the Limits of Corporate Social Responsibility, The Brookings Institution.

Waddock, S. 2000. The multiple bottom lines of corporate citizenship: Social investing, reputation, and responsibility audits, Business \& Society Review, 105(3): 323-346.

Weber, M. 2008. The business case for corporate social responsibility: A company-level measurement approach for CSR, European Management Journal, 26: 247-261.

Wernerfelt, B. 1984. A resource-based view of the firm, Strategic Management Journal, 5(2): 171-181. 
Whetten, D. A., G. Rands y P. Godfrey. 2001. What are the responsibilities of business in society? en A. Pettigrew, H. Thomas y R. Whittington (comps.), Handbook of Strategy and Management, Londres, Sage.

Winn, M. I., Kirchgeorg, M., Griffiths, A., Linnenluecke, M.K. y E. Gunther. (2010). Impacts from Climate Change on Organizations: a Conceptual Foundation. Business Strategy and the Environment, 20, 157-173. 
Apéndice 1

Bloques del instrumento de investigación

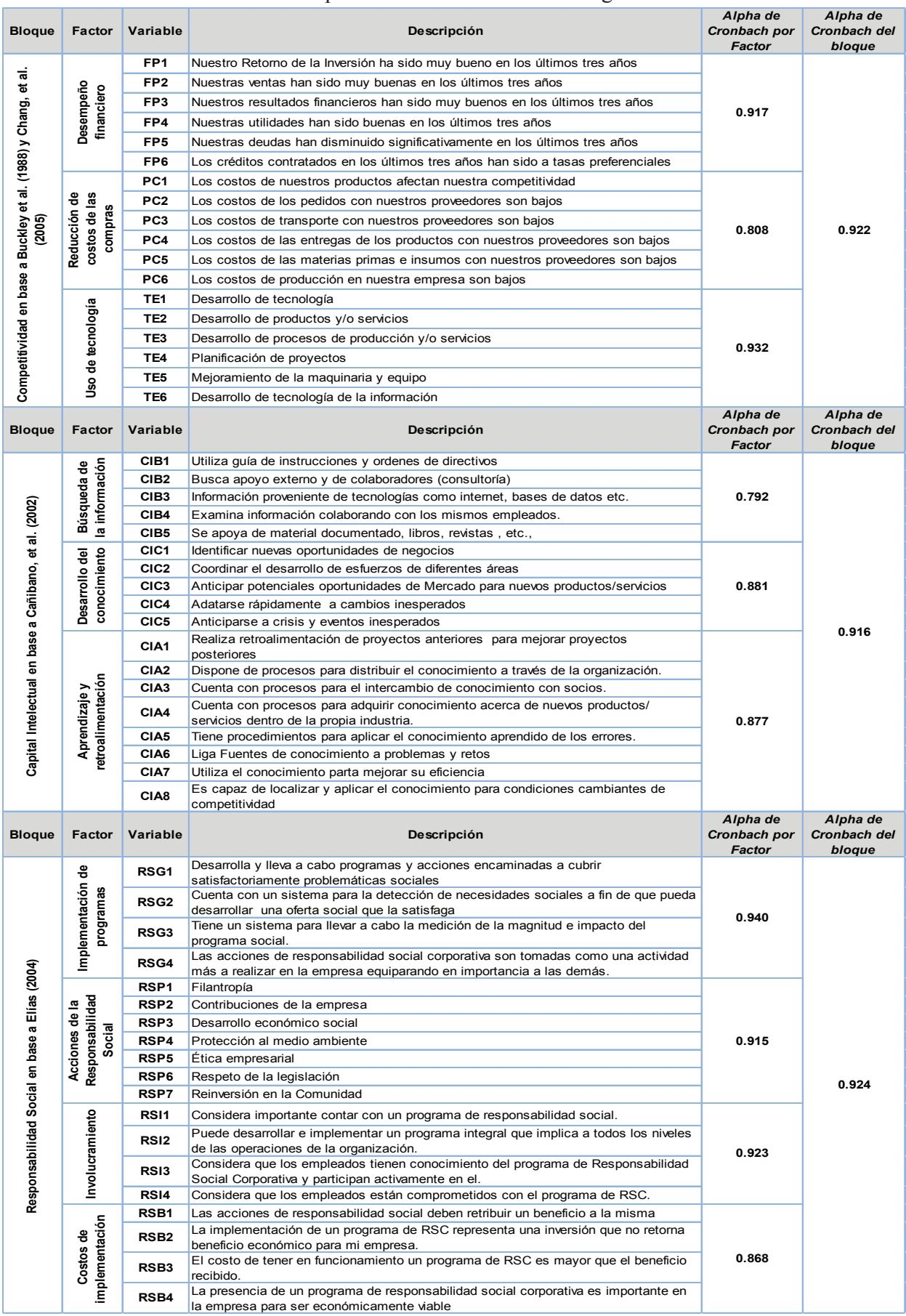

Fuente: Elaboración propia con base en los autores descritos en cada bloque.

ISSN 0188-266X - Revista de Economía - Vol. XXXI - Núm. 83 